\title{
Contrapúblicos en YouTube: el caso del colectivo trans
}

\section{Counterpublics on YouTube: The case of trans communities}

\author{
Iolanda Tortajada; Antonio-Alfredo Caballero-Gálvez; Cilia Willem
}

Cómo citar este artículo:

Tortajada, Iolanda; Caballero-Gálvez, Antonio-Alfredo; Willem, Cilia (2019). “Contrapúblicos en YouTube: el caso del colectivo trans". El profesional de la información, v. 28, n. 6, e280622.

https://doi.org/10.3145/epi.2019.nov.22

Artículo recibido el 03-05-2019

Aceptación definitiva: 30-07-2019

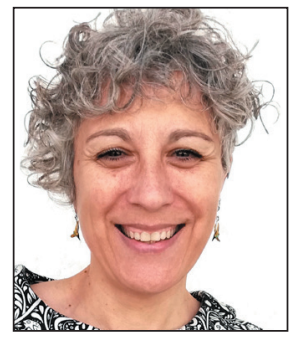

Iolanda Tortajada $\triangle$

https://orcid.com/0000-0002-9310-652X

Universitat Rovira i Virgili

Departament d'Estudis de Comunicació

Av. Catalunya, 35.

43002 Tarragona, España

iolanda.tortajada@urv.cat

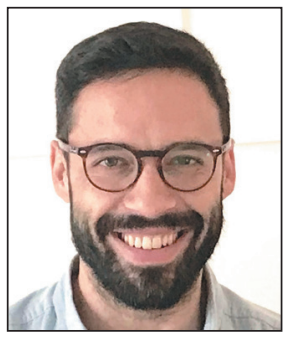

Antonio-Alfredo Caballero-Gálvez

https://orcid.com/0000-0002-8566-7853

Universitat Rovira i Virgili

Departament d'Estudis de Comunicació

Av. Catalunya, 35

43002 Tarragona, España

antonio.caballero@urv.cat

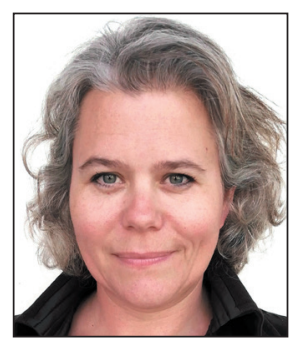

\author{
Cilia Willem \\ https://orcid.com/0000-0002-8272-498X \\ Universitat Rovira i Virgili \\ Departament d'Estudis de Comunicació \\ Av. Catalunya, 35 \\ 43002 Tarragona, España \\ cilia.willem@urv.cat
}

\section{Resumen}

La accesibilidad y democratización de los medios online ha permitido que emerjan voces alrededor de temas con escasa visibilidad. Este estudio se centra en el colectivo trans. Mientras que los públicos se generan en los medios convencionales, los contrapúblicos surgen desde los márgenes, generando espacios alternativos para debatir y discutir. YouTube se ha erigido como un espacio de participación y aprendizaje colaborativo, que rompe con los límites entre lo público/ privado y lo colectivo/personal. Tomando como estudio de caso dos youtubers trans, Elsa Ruiz Cómica y Alejandro P. E., pretendemos entender cómo se configuran sus contrapúblicos, así como sus estrategias de apropiación tecnológica y activismo. Gracias al debate generado por sus vídeos, estos youtubers trans consiguen trasladar conceptos innovadores a un público más amplio, contribuyendo al reconocimiento de identidades (sexuales) diversas, y el cuestionamiento del binarismo de género.

\section{Palabras clave}

Contrapúblicos; YouTube; Trans; Transexualidad; Identidad; Género; Democratización mediática; Comunicación; Medios sociales; Audiencia.

\section{Abstract}

The accessibility and democratization of online media has allowed voices to emerge around issues with low visibility. In this study we are looking at the trans community. Whereas publics are generated in mainstream media, counterpublics

Financiación

Este artículo se enmarca en el proyecto RESPECT- Produsage juvenil en las redes sociales: construcción de la identidad sexual y gestión de las desigualdades de género, con referencia FEM2017-83302-C3-1-P. Financiado por el Ministerio de Economía, industria y competitividad. 
emerge from the margins, generating alternative spaces for debate and discussion. YouTube has emerged as a space for collaborative learning and participation, breaking the boundaries between public/private and collective/personal. Taking Elsa Ruiz Cómica and Alejandro P. E.'s YouTube channels as a case study, we intend to understand how their respective counter-publics are configured, and to analyze their strategies of technological appropriation and activism. Thanks to the debate generated by their videos, these trans youtubers manage to transfer innovative concepts to a wider audience, contributing to the recognition of diverse (sexual) identities, and to the questioning of gender binarism.

\section{Keywords}

Counterpublics; YouTube; Trans; Transsexuality; Genre; Transgender; Identity; Media democratization; Communication; Social media; Audience.

\section{Introducción}

En las primeras teorizaciones sobre internet (Castells, 1997) ya se reflejaba una preocupación política, administrativa y académica por las diversas brechas digitales que se detectaban en la evolución de la sociedad informacional, y un consenso sobre la importancia de dar a todo el mundo oportunidades y capacitación para crear contenido en la Red (Bartolomé-Pina, 1996; Buckingham, 2007).

La aparición de internet y de las redes sociales permite que las audiencias ejerzan prácticas mucho más deliberativas y relacionales, acercándose a lo que habitualmente ha sido definido como 'públicos' y difuminando la separación entre ambos conceptos, así como las esferas de lo privado y de lo público, que ahora se entremezclan (Masip; Ruiz-Caballero; Suau, 2019; Papacharissi, 2010). Lo privado no sólo se expone públicamente, sino que forma parte del contenido a debatir. Las identidades, si bien ya formaban parte de lo político (Giddens, 1995) cobran un peso fundamental en las redes sociales, ya que éstas canalizan las estrategias de gestión del yo, cada vez más trascendentales para la deliberaciónn política. Así, las redes sociales no son sólo el espacio en el cual se mediatizan los públicos, sino el entorno en el que los temas de debate, más que nunca, parten del deseo y la reivindicación de construir el yo desde la propia voz (Beck, 1998).

Este artículo analiza un conjunto de prácticas online de dos youtubers trans españoles que, desde una acción de base fundamentalmente política, consiguen que un espacio público se convierta en una esfera pública (Pa-

pacharissi, 2002; Travers, 2003). En este proceso de conversión, la participación de los contrapúblicos es imprescindible. Los contrapúblicos, como los públicos, no son organizaciones formales, sino que se constituyen mediante la circulación de textos y de la interacción en contextos específicos (Renninger, 2014). Sin embargo, a diferencia de los públicos, los contrapúblicos surgen en los márgenes, en la oposición a lo hegemónico (Fraser, 1990; Warner, 2002). Por tanto, los contrapúblicos son intrínsecamente espacios políticos.

El objetivo principal de este estudio es entender cómo se configuran los contrapúblicos, en este caso trans, que generan espacios de debate y de cuestionamiento de lo establecido y se convierten progresivamente en productores y distribuidores de nuevo contenido (Shifman, 2011). Hemos escogido YouTube como plataforma para el análisis, ya que se ha consolidado en los últimos años como un espacio de participación cultural y de diversidad (Burgess; Green, 2009) en el que aquellos colectivos que quieren trascender lo comercial y lo normativo han podido desarrollar su activismo desde una lógica propia que tiene un gran interés social y académico:

- interés social porque, sólo desde su posición subordinada, pueden estos grupos hacerse visibles (Travers, 2003), ofrecer entornos virtuales seguros desde los que construir una comunidad (Jenzen, 2017; Warner, 2002) e impulsar debates que llegan al público mayoritario (Travers, 2003; Trejo-Delarbre, 2009; Renninger, 2014; Jackson; Foucault-Welles, 2015);

- interés académico porque son un ejemplo relevante de cómo operan los contrapúblicos virtuales y de cómo sus aspiraciones se hacen centrales y tienen un impacto en lo cotidiano (Ellison; Boyd, 2013; Renninger, 2014; Wargo, 2017).

En este sentido YouTube es un espacio de prácticas novedosas y versátiles (Trejo-Delarbre, 2009), en el que grupos particulares tienen la posibilidad de crear nuevos géneros. En el caso concreto del mundo trans, Raun (2010) ha tipificado el material en YouTube como principalmente vídeos autobiográficos, de corta duración, producidos mayoritariamente por jóvenes, con una narrativa y estética identificable y dirigidos a una audiencia concreta. El contenido de estas producciones tiene que ver principalmente con la exhibición de cuerpos subalternos que rompen con la heteronormatividad y con el plano visual binario (Álvarez-Uría; Platero-Méndez; Rosón-Villena, 2014; Halberstam, 2018). La presentación y reivindicación de su cuerpo, permite a los youtubers trans romper con los estereotipos que ofrecen los medios tradicionales acerca de lo trans, reinventarse y repensarse continuamente para reemplazar determinadas construcciones del género (Alexander, 2002; Raun, 2010; Rosen, 2015).

\author{
El activismo propio de YouTube es clave \\ para la configuración tanto de identida- \\ des como de comunidades, así como la \\ generación de un contrapúblico en red
}


La producción trans en YouTube, pues, ha sido leída como un acto íntimo, terapéutico y político (Raun, 2010; 2016; Horak, 2014; Barnett, 2015; Cavalcante, 2016; Jenzen 2017). Por un lado, las narrativas de la intimidad se consolidan como práctica mediática en la que las redes sociales juegan un papel crucial en la creación y difusión de autopresentaciones que dan sentido al género, la sexualidad y el deseo (De-Ridder; Van-Bauwel, 2015). Por otro lado, este énfasis en lo íntimo está fuertemente conectado con la reivindicación política. La mayoría de youtubers trans, en España y fuera, conciben sus prácticas mediáticas personales como una 'narración biográfica ininterrumpida' mediante la cual las definiciones tradicionales y estereotipadas del género son sustituidas por otras propias, que se encarnan en el cuerpo y en su tránsito (Raun, 2010) y que permiten cuestionar las convenciones (Barnett, 2015). En un ejercicio de reflexividad que se extiende al cuerpo (Beck, 1998) y que se articula online (Cavalcante, 2016), esta exposición personal es un proyecto de recuperación y de pertenencia en el que darse a conocer de manera diferente (Jenzen; Karl, 2014; Wargo, 2017; Cavalcante, 2016). Otro de los aspectos interesantes de este tipo de producción es que el cuerpo trans no busca ser observado o conocido, sino que pretende cuestionar todos los cuerpos (Halberstam, 2018); un ejercicio en el que, a través de la exposición de corporalidades diversas en YouTube se produce una reinvención empoderadora (Raun, 2010).

En resumen, nuestro punto de partida es éste: la posibilidad por parte de los usuarios de controlar la representación del cuerpo subalterno a través de la imagen en movimiento convierte YouTube en un sitio por excelencia para

- la apropiación tecnológica y el activismo trans;

- la configuración tanto de identidades como de comunidades (Cavalcante, 2016, Jenzen, 2017);

- la generación de un contrapúblico en red (Jackson; Foucault-Welles, 2015).

\section{Metodología}

El contrapúblico trans polemiza de forma más o menos rupturista sobre el binarismo de género que predomina en la sociedad y en la representación cultural. A pesar de su heterogeneidad, el colectivo trans -invisibilizado por los medios mainstream - comparte el propósito de dar visibilidad al tránsito y a las identidades que de él se derivan y utilizan las redes sociales, de forma activa e intensa, para lograrlo. Estudiar los casos de los youtubers Elsa Ruiz Cómica (MTF: male to female, 17.441 suscriptores en abril 2019) y Alejandro P. E. (FTM: female to male, 66.970 suscriptores en abril 2019) es relevante para entender cómo se configuran los contrapúblicos alrededor de lo trans en España, y cómo se construyen los espacios de debate y de cuestionamiento de lo establecido sobre la identidad de género en nuestro país. Para ello, y después de revisar la producción completa de los canales de Elsa Ruiz Cómica y Alejandro P. E., hemos realizado un close reading de un conjunto de 8 vídeos, escogidos por su temática, que fueron publicados entre septiembre de 2016 y noviembre de 2018 y cuya duración media es de 10 minutos y medio (en el caso de Elsa) y de 6 minutos (en el caso de Alejandro P. E.). Además, hemos realizado un análisis cualitativo de discurso con enfoque crítico (Van-Zoonen, 1994) de los 200 comentarios expresados sobre esas piezas y de las 709 respuestas que los comentarios han provocado con el propósito de recoger algunos de los principales debates que se generan sobre las expresiones de género, la transición y el cuerpo, ya sean reproductores o contrahegemónicos (Kellner; Kim, 2010).

\section{Aportaciones de los contrapúblicos trans}

Siguiendo a Fraser (1990), las producciones trans permiten la aparición de contrapúblicos trans. Analizando los casos de Elsa Ruiz Cómica y Alejandro P. E., el potencial emancipador de los contrapúblicos se refleja en las siguientes características:

\section{Creación de conceptos que denuncian la desigualdad o la discriminación}

Tanto Elsa como Alejandro, aunque cada uno desde una perspectiva diferente, dedican parte de su narrativa al activismo y a la denuncia a favor del colectivo trans. Como en el caso de los contrapúblicos feministas (Fraser, 1990), en los vídeos trans se introduce un conjunto de conceptos que permiten poner de manifiesto los procesos de invisibilización y discriminación que sufre el colectivo y que dotan a la sociedad de herramientas para comprender y reconocer la diversidad sexual.

Un tema crucial en la configuración de los debates sobre identidades políticas trans es la autodefinición.

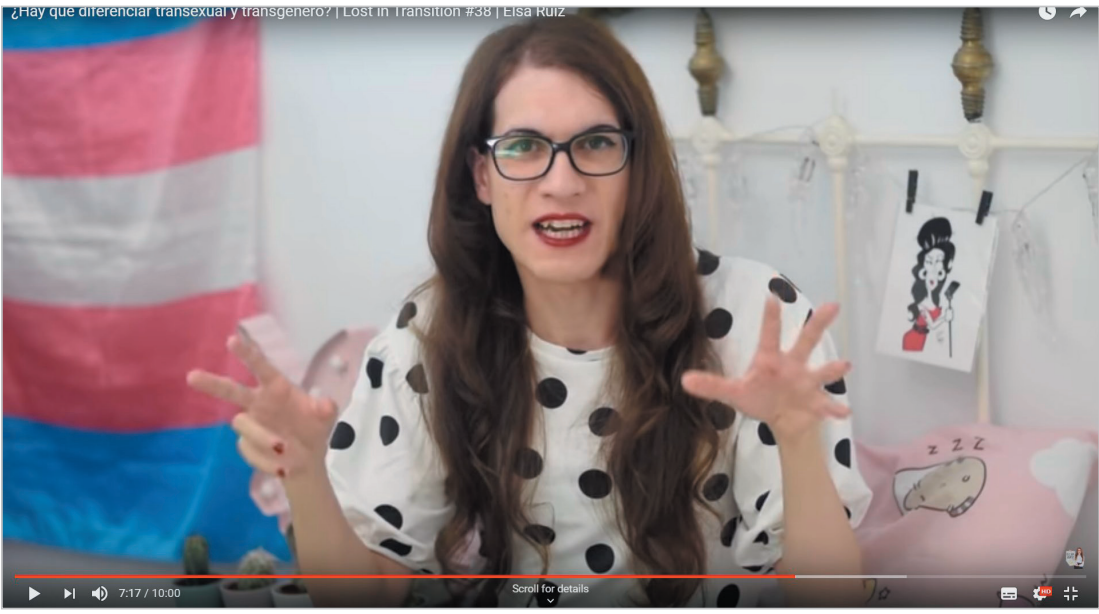

Imagen 1. ¿Hay que diferenciar transexual y transgénero? https://www.youtube.com/watch? $v=g Y C Z H I 5 Q$ stw $\& t=428 \mathrm{~s}$ 
En ¿Hay que diferenciar transexual y transgénero? (imagen 1) Elsa defiende el uso de trans como concepto inclusivo que ha de servir, en primer lugar, para evitar posibles comparaciones entre los procesos de cada persona y, en segundo lugar, para que lo transexual o la cirugía no se consideren como algo 'mejor' que la opción de no modificar el cuerpo. Reivindica que no hay una forma única de ser trans y denuncia las presiones legales y médicas que les empujan a seguir una vía de tránsito estrecha. Ser trans, según esta youtuber, es un 'traje a medida'. Aboga por la solidaridad entre personas trans, porque no se generen distinciones dentro del propio colectivo y que las personas trans no binarias tengan un espacio de reconocimiento. Para ella cualquier tipo o manera de ser trans es perfectamente válida. Desde esta apuesta reflexiva (Giddens, 1995), la identidad trans que se propone en los vídeos de Elsa trasciende el yo particular y eleva el debate a lo político desde la centralidad del cuerpo (Beck, 1998).

En los comentarios abundan las muestras de agradecimiento por los vídeos y las expresiones de acuerdo y satisfacción con los contenidos. Un punto interesante es que en las reflexiones que se generan también intervienen personas que no se definen como trans, lo que apunta a que, aunque sea de forma incipiente, el debate supera el espacio del contrapúblico. Quienes participan en este espacio tratan de ponerse de acuerdo acerca de algo en el mundo (Habermas, 1987) para reivindicar derechos y deconstruir las formas de etiquetar que discriminan. Además, el canal aparece como un espacio en el que refugiarse: "Necesitaba este vídeo hoy. Muchas gracias Elsa, de corazón” (1EC5). El contrapúblico trans aparece como una comunidad que comparte ideas y sentimientos y que se apoya, también con humor:

"como dijo la hierbas de LQSA (La que se avecina) (cosa que comparto totalmente): nos enamoramos de almas, no de genitales" (1EC21).

Finalmente, las muestras de empatía y solidaridad son constantes:

"no soy trans y no me puedo imaginar la fortaleza que hay que reunir para luchar constantemente contra los prejuicios de la sociedad" (1EC1).

En ¿Es bueno el cispassing? (imagen 2) Elsa introduce el concepto de cispassing (el 'pasar' por completo por tu género deseado), criticando que la aceptación social de las personas trans tenga que ver con un aspecto que las asimila a un género o a otro, que las normaliza y que, por ello, las hace menos incómodas. Además, el cispassing margina a las personas trans no binarias, lo cual según Elsa, es un problema: lo considera una discriminación presuntamente positiva que, en realidad, es la imposición de una apariencia concreta. El problema es de la sociedad, no del nivel de passing

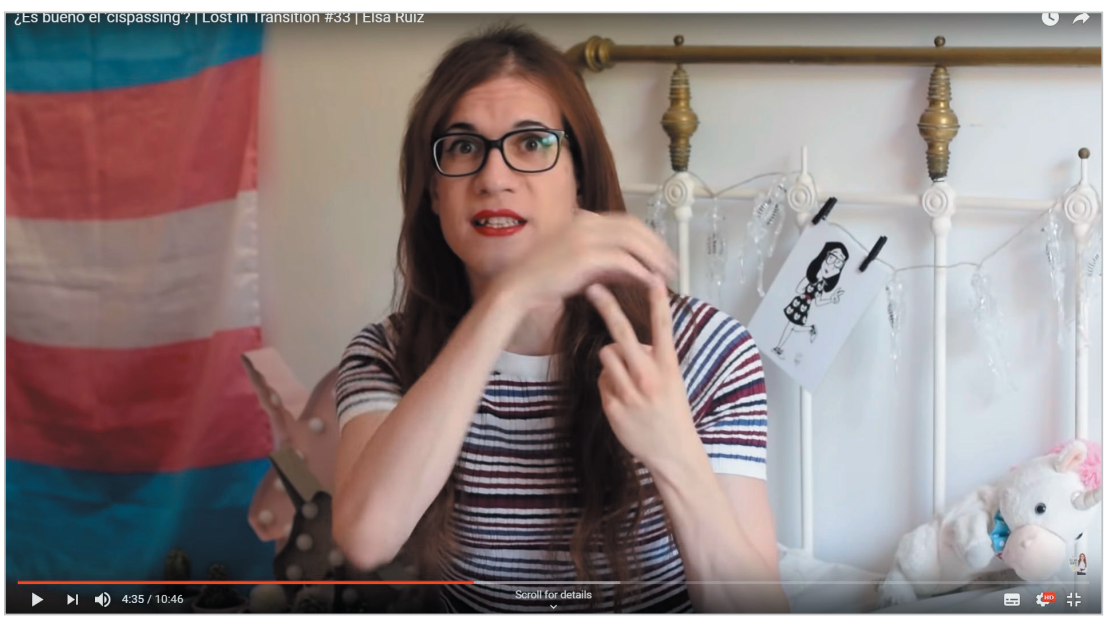

Imagen 2. ¿Es bueno el cispassing?

https://www.youtube.com/watch?v=cyJKiB8ESXA\&t=211s

de cada persona trans, pero, para Elsa, es importante no premiar el cispassing y rechazar los comentarios que refuerzan la idea de que lo que está bien es que 'no se note que eres trans'. El debate entre el público de Elsa se construye como un momento de autoafirmación:

“Creo que jamás voy a saber cuál es mi género pero últimamente he comprendido que no es necesario y estoy aprendiendo a que me dé igual. Es hora de ser une misme" (3EC2).

y de reconocimiento:

“Creo que gracias a gente como tú y a lo que está evolucionando la sociedad, aunque sea a muy pequeños pasos, cada vez será más fácil que cada cual se exprese con la ropa que quiera o haga lo que le apetece. Así que me alegro de que cada vez las nuevas generaciones tengan información y sean más abiertas" (3EC11).

"No tenemos por qué ajustarnos al patrón binario hetero-normativo, que cada cual sea y desee como le dé la gana!" (3EC13).

Hay un gran acuerdo entre el contrapúblico de Elsa y sus planteamientos:

"iSi, soy trans, he tenido esa suerte! ... El día que intentemos dejar de tener cispassing, seremos aceptades como una opción más. Sin más" (3EC15),

aunque también se exponen las diferentes ventajas del passing :

"es la tranquilidad de no ser blanco de miradas confundidas, de caminar tranquilamente por la calle sin escuchar insultos o temer por tu vida" (3EC22).

Como en el resto de vídeos, las muestras de respeto y agradecimiento son continuas. Es una admiración de fans: 
“Es oficial ESTE ES MI VIDEO FAVORITOOOOOOO pero si es que te adorooooo me encanta pensar CONTIGO” (3EC19),

cargada de contenido político:

"yo la respeto porque ella [su hija] no quiere ser visible, pero te doy las gracias por tu visibilidad. Es muy bueno que se vea la visibilidad, aunque ella no quiere" (3EC23).

\section{Ruptura vs. confirmación del binarismo de género en los debates online}

Muchas de las reflexiones que se plantean en las producciones de Elsa y de Alejandro tienen que ver con la dificultad de construir identidades y cuerpos en sociedades cisnormativas en las que lo queer aparece como lo imposible e incategorizable (Butler, 2007). Esto lleva a posiciones esencialistas, como las de Alejandro P. E., y a apuestas por lo fluido, como las de Elsa. Los argumentos empleados por Elsa buscan el reconocimiento de las personas que no se sienten identificadas con ninguno de los dos géneros y romper con aquellos parámetros que tradicionalmente definen los cuerpos (Butler, 2007). La exacerbación o la ruptura del binarismo de género están en el centro del debate.

En Nunca seré como un chico normal (?) o sí (imagen 3) la autodefinición que hace Alejandro toma como referencia un modelo masculino hegemónico que le aleje al máximo de cualquier identificación femenina. En este vídeo, Alejandro denuncia:

"Recibo constantemente mensajes destructivos, o intentando ser destructivos, mencionando siempre lo mismo: que nunca seré hombre por el hecho de que mi cromosoma es XX o porque no tengo un rabo entre las piernas".

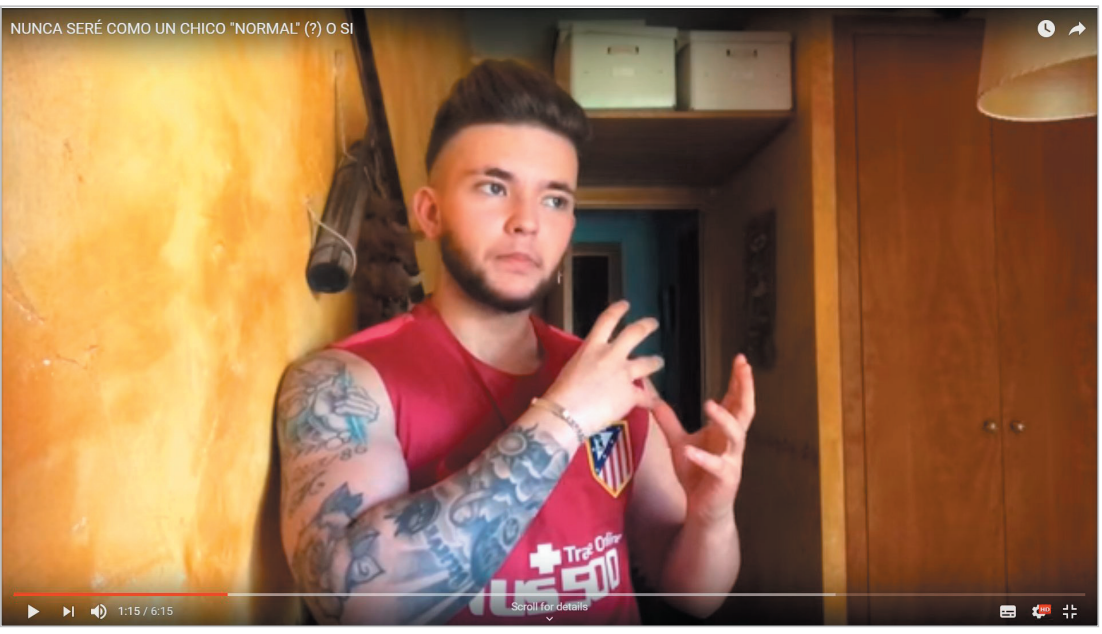

Imagen 3. Nunca seré como un chico normal (?) o sí

https://www.youtube.com/watch?v=Huz8jOUuZB4\&t=13s

Para defender su virilidad, Alejandro comienza el vídeo con una crítica hacia todos aquellos hombres que de alguna u otra manera hacen daño a las mujeres a través de acosos, agresiones o violaciones. Una vez ha dejado claro que él es mucho más hombre que todo aquel que hace daño a mujeres y/o niños, afirma que su virilidad no se define por su construcción genética, es decir, no se basa en cromosomas ni en su genitalidad, sino en su físico:

"Yo soy consciente de que tengo mis limitaciones como chico trans, no me podré ver como chico cisgénero, pero no por ello voy a dejar de luchar".

Alejandro defiende que la transexualidad puede ser 'ocultada' a través de la apariencia física. Para ello, muestra un catálogo de modelos transexuales FTM -female to male-, que responden al modelo masculino hegemónico al que él aspira: cuerpos jóvenes, sanos y musculados. Todos ellos chicos trans (de apariencia $100 \%$ masculina), lo que quiere dar a entender que 'a pesar de ser trans' son hombres y la sociedad al ver sus fotos no piensa que son chicos trans. Alejandro así también defiende un modelo heterosexual, alegando que lo que ocurre es que muchos chicos cisgénero tienen miedo de que los chicos trans puedan ser mucho más atractivos que ellos:

"No pasa nada, no nos vamos a quedar con todas las mujeres del mundo... Aunque seríamos capaces".

Todos los comentarios analizados de este vídeo muestran apoyo a Alejandro P. E.: existen mensajes de admiración como el comentario:

“Alex, para mí, eres admirable, guapo, lindo, simpático y único. Yo encantada contigo. Ya te amo!!!!” (1AC24).

También existen comentarios que apoyan su argumento sobre la disociación de la genitalidad con el género, tal y como aparece en los comentarios:

"Alexito no necesitas tener algo entre las piernas para que pienses que eres un hombre, para mi lo eres. Un caballero como vale oro además está guapísimo" (1AC19)".

Hay sólo tres comentarios que son críticos en cuanto al modelo masculino defendido por Alejandro P. E. y al que, tal y como él mismo reconoce, aspira. Estos comentarios se centran en poner en duda la relación entre musculación y masculinidad:
Las reflexiones de las producciones de Elsa y Alejandro P. E. tienen que ver con la dificultad de construir identidades y cuerpos en espacios normativos 
"Alex te apoyo siempre, pero creo q aquí te has centrado demasiado en el aspecto físico. Un hombre trans no tiene porque ser el prototipo de hombre musculoso para ser más o menos hombre. Eso también es un estereotipo creado por la sociedad. Un abrazo!" (1AC9)

e incluso un comentario donde se recrimina que la vinculación entre transexualidad y hormonación:

"Suelo estar de acuerdo con todo lo que dices pero tengo que decir que con una cosa no. En mi opinión un chico trans que no se hormone, que tenga pecho, pelo largo... Sigue siendo hombre [...]" (1AC2).

Como la mayoría de los transexuales FTM, Alejandro P. E. se preocupa mucho por el uso de la testosterona. En el vídeo ¿Cómo era antes de la testosterona? Timeline (imagen 4) muestra el cambio físico que ha desarrollado en los últimos tres años.

Los comentarios a este vídeo son positivos: de nuevo la empatía por parte de los seguidores del youtuber es la base de los mensajes dedicados a sus vídeos. Se trata de mensajes en los que se ponen de manifiesto los grandes cambios logrados por Alejandro P. E., como:

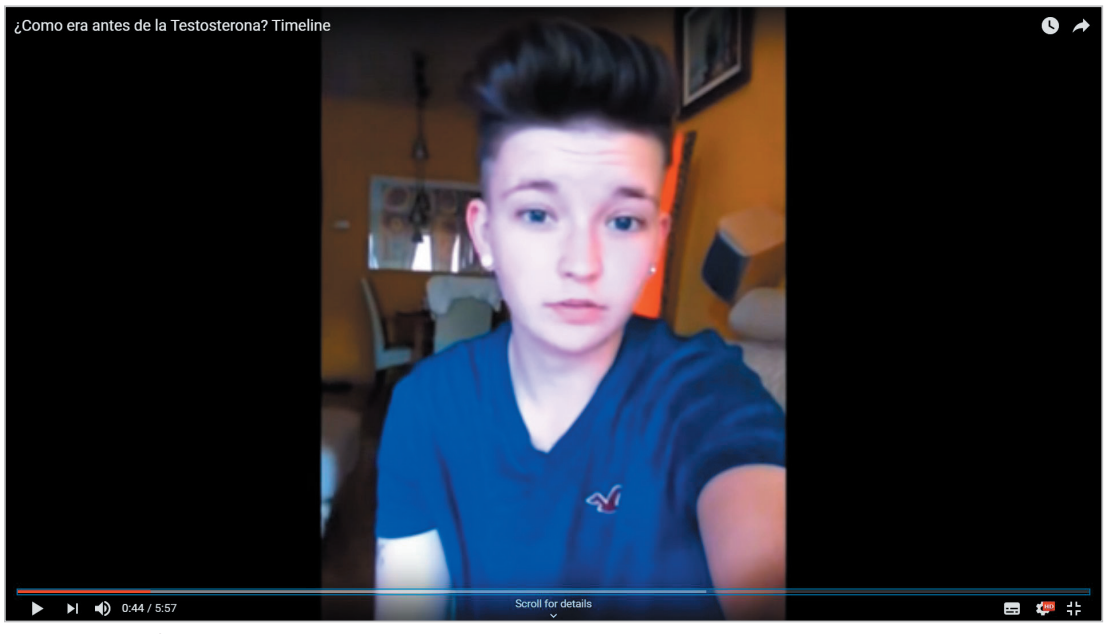

Imagen 4. ¿Cómo era antes de la testosterona? Timeline https://www.youtube.com/watch?v=Z6qX-Knsktc\&t=253s

"Tu cambio es impresionante" (AC18)

"Ya te digo Alex, cambio no, cambiazo. Además que ahora eres super super masculino" (AC12).

Una gran parte de estos comentarios se refieren a la voz, uno de los aspectos con los que Alejandro aún no está del todo satisfecho, ya que espera poder tener una voz más grave:

"Pues a mí me gusta mucho tu voz, me parece sexy" (AC2)

"iQué bien hablas! Ah y tu voz ya está maravillosa, enamora" (AC7)

"Tienes una voz muy bonita!!!! esta perfecta!!" (AC19).

Destacan tres comentarios de hombres cisgénero que se suman a los alegatos de admiración a los cambios de Alejandro, como serían:

"Yo soy hombre biológico y tu voz suena perfecta y más grave y masculina que la mía" (AC1)

"Parece mas hombre que yo. Me encantó su cambio" (AC23).

Desde un posicionamiento antagónico al defendido por Elsa, Alejandro P. E. en ¿Me opero o no? ¿Hijos biológicos? https://www.youtube.com/watch?v=uJMP64_L1XO

se aleja de toda la ambigüedad que se pueda desprender de su condición trans, para identificarse con un cuerpo cis masculino, defendiendo una paternidad heteronormativa representada por los binomios padre/hombre y madre/mujer. Alejandro comienza a atacar otras opciones trans:

“No sería el típico chico trans tan moderno que se queda embarazado él [...] Respeto que algunos chicos se quieran quedar embarazados pero no lo entiendo".

Este comentario le conduce a posicionarse en contra de parte del colectivo trans:

"Lo peor de ser trans no son las operaciones sino los trans tocacojones, es lo peor de este colectivo [...] son muy pesados o 'pesades' como les gusta llamarse a ellos, en fin".

En este vídeo, los comentarios y respuestas se centran en primer lugar en dar respuesta y opinar a la cuestión planteada por Alejandro P. E. sobre la histerectomía (extracción del útero). El resto de comentarios se dividen en los que expresan su admiración por el proceso de transición de Alejandro, resaltando sus cualidades físicas y unos pocos comentarios que critican la forma en que el youtuber se dirige al resto del colectivo trans que no piensa como él mismo:

“Pero a ver, que seas trans no significa que tengas que ser 'radical', yo por ejemplo soy amante de los animales y no te voy a decir de todo porque comas carne [...]" (3AC10)

"La terminación neutra también es necesaria por la cantidad de géneros que hay que no son ni chicos ni chicas y para que en el habla cotidiana no se os represente sólo a los hombres. Así que no entiendo esas burlas" (3AC19). 
En el vídeo Dejo la transexualidad

https://www.youtube.com/watch?v=xUYVBhsFNas\&t=307s

al cual hace referencia Elsa, Alejandro define el mundo de la transexulidad como un "circo". El youtuber reconoce que inició su canal para mostrar y explicar sus cambios en su transición. Sin embargo, considera que esa época ya ha pasado, y él no quiere estar dentro de un colectivo que le "avergüenza". Comenta varios casos en los que otros/as youtubers trans han jugado con la disforia de género. Define la comunidad trans como un mundo "envidioso y miserable".

Se trata de uno de los vídeos más problemáticos y criticados del youtuber. La mitad de los comentarios son de apoyo, y el resto critican duramente las afirmaciones realizadas por Alejandro en el vídeo analizado. En sus mensajes de apoyo, hay comentarios que le defienden:

"Haces bien porque sinceramente a muchos les atrae el morbo de la transexualidad, bienvenida la nueva temática de tu canal es lo mejor que has hecho [...]"

y algunos que de nuevo resaltan sus cualidades físicas:

"Te veo súper, súper guapo y muy atractivo. Enhorabuena (4AC10)".

Sin embargo, son los comentarios críticos con el youtuber los que reciben un mayor número de respuestas y debate. Los comentarios que destacan siguen la línea argumental defendida por Elsa en respuesta a este vídeo:

"Hay gente trans que acepta que hay cuerpos que no se corresponden a la cisnorma y que pueden quererse a sí mismo. Si tu quieres ser el prototipo de machito es tu problema pero un chico trans puede disfrutar y mostrar su cuerpo si le da la puta gana" (4AC3).

Algunos comentarios caen en el insulto:

"Qué capullo... hay que seguir algún patrón para ser el trans perfecto? Cada uno lo lleva como puede y lo más importante como quiere, por mí, quítate el canal, has dejado claro cómo eres" (4AC10).

Estamos ante un ejemplo claro de cómo los contrapúblicos no sólo se manifiestan en los canales donde están suscritos sino que interactúan en distintos canales, y cómo se multiplica la repercusión de sus vídeos, cuando otros youtubers se hacen eco de sus producciones. En este caso, es el vídeo de Elsa el que cuestiona el vídeo de Alejandro, generando una actividad mucho mayor en el vídeo de Alejandro si la comparamos con otros tres vídeos anteriores analizados.

En ¿“"Dejar" de ser trans?

https://www.youtube.com/watch?v=phRXaC50svU\&t=51s

Elsa responde directamente a un vídeo que ha colgado Alejandro (Dejo la transexualidad). Así, este vídeo se plantea como un debate, un espacio en el cual contraargumentar las afirmaciones de Alejandro sobre las "cosas del mundo trans". Para ello, Elsa contesta al esencialismo del discurso de Alejandro defendiendo que necesitamos toda una vida para ser quienes somos y cuestionando que, desde nuestro nacimiento, se nos imponga quiénes somos y no nos dejen tomarnos nuestro tiempo y decidirlo. Nos explica que ella empezó su transición hace cuatro años, a los 27 . No cree que haya un momento vital adecuado y defiende que no hay una única manera de hacer el tránsito:

“'La manera de ser trans es correcta si a vosotres [Elsa usa la e como fórmula inclusiva] os vale'. Ser trans, para ella, es conocerse. Las personas trans son una muestra de la riqueza humana. Usa una metáfora que ha popularizado ella misma, la de que las personas no somos diferentes de las pizzas: los ingredientes pueden ser muchos y variados y que 'el secreto está en la masa'”.

Buena parte de los comentarios agradecen a Elsa la claridad, la coherencia y la fuerza con la que explica. Celebran "las imágenes mentales que crea" (4EC6), especialmente la de la pizza:

"yo soy una pizza con piña mental" (4EC1),

"no he encontrado a dos pizzas iguales" (4EC1) o

"soy una pizza con tantas cosas que ni siquiera sé qué tipo de pizza soy" (4EC3)

son algunos ejemplos. En el debate participan personas que se definen como chicas transgénero, personas en transición mental por ahora, trans gays sin disforia, chicas cis o alguien no trans. Tanto ellas como el resto de participantes creen que su canal es un espacio pedagógico y de empoderamiento, y reconocen constantemente el valor de este espacio:
Los canales de Elsa y Alejandro P. E. dotan a la sociedad de herramientas para comprender y reconocer la diversidad sexual

"Qué tía tan coherente y sana. Dice lo que piensa con absoluto respeto y siempre intentando educar. Hace falta más gente así, no en el colectivo, sino en la sociedad en general." (4EC23)

"Olé! me encanta tanto tu amplitud de miras..., tus opiniones son un buen trato a la humanidad! Gracias por expresarte, alivia un montón saber que existe gente como tú" (4EC20). 
En este caso, todos los mensajes comparten lo expresado por Elsa, a la vez que enriquecen el debate con experiencias propias.

\section{Las identidades individuales y colectivas como proyecto político}

Los y las youtubers trans tratan temas y preocupaciones que se debaten en la vida real y que definen la identidad personal y del colectivo. Al trasladarlos online amplifican sus repercusiones.

\section{En ¿Por qué no me hormono?}

https://www.youtube.com/watch?v=6eFMFOzpQQU

Elsa trata de desmontar la correlación prejuiciosa entre la hormonación y el tránsito, denunciando las presiones legales y médicas que vinculan el reconocimiento oficial a la transformación forzosa del cuerpo y defendiendo que, para la transición, no hay protocolos, sino trayectorias.

En el debate, además de alabar la fortaleza de Elsa y subrayar su carisma, se expresa el acuerdo con que "La transición es un traje a medida" (2EC1) y que hay múltiples maneras de ser. Se reflexiona sobre la importancia de que cada quien decida sobre su transición, más allá de las hormonas, de la cirugía y de la apariencia. Tanto el vídeo como los comentarios ponen de manifiesto que estos discursos basados en la experiencia son un proyecto de recuperación y de pertenencia (Jenzen; Karl, 2014; Wargo, 2017; Cavalcante, 2016) desde el cuestionamiento de todos los cuerpos (Halberstam, 2018).

Dos de los comentarios reciben algunas respuestas transfóbicas. Las respuestas son menos reflexivas que los comentarios y, en ocasiones, consisten en conversaciones de dos en los que ambas personas se atacan pero no dialogan. El 2 EC4 recibió 50 respuestas. El debate sobre el lenguaje inclusivo que se generó no sirvió para acercar posturas ya que muchos mensajes no eran argumentativos y se partía de posiciones muy definidas. En ocasiones se insulta o se menosprecia al otro, así como los puntos de vista con los que no se está de acuerdo que se consideran inferiores, menos inteligentes o depravados. Curiosamente, el desencuentro se produce por cuestiones lingüísticas, pero no identitarias.

En general se apuesta por reflexionar y actuar, cambiando los valores propios:

"para ser trans no necesitas odiarte, ni a tus genitales" (2EC8)

y los de la sociedad:

"hay que renovar lo que significa ser hombre, mujer o ninguno" (2EC8).

También se aprovecha para cuestionar las presiones, ya sean autoimpuestas o sociales:

"muchos deberían dejar a cada una tomar sus decisiones y no establecer una lista de sí y no en qué hacer durante una transición (2EC12).

Finalmente, muchos de los comentarios son para felicitar a Elsa y agradecer todo lo que reciben al verlos:

"Me ha encantado tu vídeo, es el primero que veo y nunca me había sentido tan identificado" (2EC22)

"Felicitaciones y gracias por disipar prejuicios y tanta desinformación" (2EC23).

Esto demuestra la importancia de los contrapúblicos para la conformación paralela de identidades y comunidades en internet (Cavalcante, 2016; Jenzen, 2017).

\section{Conclusiones}

Dando respuesta al objetivo marcado, el análisis demuestra cómo la relación de los youtubers con sus contrapúblicos se articula a partir de la libertad a la hora de expresarse, la ausencia de autocensuras, así como el apoyo, admiración y defensa generalizado a los youtubers trans a los que siguen. Hay que diferenciar entre Elsa Ruiz -que genera una relación con su público basada en el debate abierto, transversal y horizontal-y el canal de Alejandro P. E., en el que apenas encontramos respuestas del propio youtuber, y cuyo público hace comentarios que se enmarcan dentro del terreno de lo físico y superficial, dejando de lado algunas cuestiones del debate político en torno a lo trans.

El análisis de estos espacios virtuales de debate y crítica nos ha permitido conocer cómo se configuran los contrapúblicos de las comunidades trans, en este caso en España. Su doble rol de productores y distribuidores dentro del universo YouTube valida nuestro punto de partida al comprobar cómo los canales analizados han abierto las puertas a la consolidación de un espacio online. Se comprueba que YouTube es un espacio de aprendizaje colaborativo: los contrapúblicos que allí conviven aprenden unos de otros, se aconsejan, animan, critican, consultan y acompañan las transiciones de Elsa y de Alejandro, en un intercambio entre pares a través de sus canales de YouTube. Del mismo modo, llevan lo personal a lo político, forzando los límites entre lo privado y lo público, desafiando cuestiones tabú que se encuentran fuera del discurso normativo que domina la comunicación no digital. Ante este nuevo escenario, donde los youtubers son al mismo tiempo productores y gestores de contenidos de dicha cultura participativa (Jenkins; Ito; Boyd, 2016) se ha habilitado un nuevo escenario de lo íntimo (Sibilia, 2008) a partir del cual los contrapúblicos pue-

Tanto los youtubers como sus contrapúblicos llevan lo personal a lo político, forzando los límites entre lo privado y lo público 
den manifestarse y expresarse sin ningún tipo de reservas ni control. Para estos contrapúblicos, las redes sociales y las plataformas participativas ofrecen un universo autónomo y creativo en el que expresar y "representar sus propias experiencias" (Morduchowicz, 2012, p. 11). Por lo tanto, las estrategias comunicativas que emplean los youtubers a la hora de relacionarse con sus contrapúblicos se basan en la empatía, el intercambio de aprendizaje entre pares, la libertad sin censura en sus discursos, y finalmente la superación de los límites entre lo público y lo privado a partir de la exposición del cuerpo subalterno como reivindicación.

\section{Referencias}

Alexander, Jonathan (2002). "Homo-pages and queer sites: Studying the construction and representation of queer identities on the world wide web". International journal of sexuality and gender studies, n. 7, pp. 85-106.

https://doi.org/10.1023/a:1015888915258

Álvarez-Uría, Amaia; Platero-Méndez, Lucas; Rosón-Villena, María (2014). “El 'estilo de la carne' en Maikrux y Falete: feminidad, humor y agencia". Feminismo/s, n. 24, pp. 143-162.

https://doi.org/10.14198/fem.2014.24.07

Barnett, Joshua T. (2015). "Fleshy metamorphosis: Temporal pedagogies of transsexual counterpublics". En: Spencer, Leland; Capuzza, Jamie (eds.). Transgender communication studies: Histories, trends, and trajectories. London: Lexington Books, pp. 155-172. ISBN: 9781498500050

Bartolomé-Pina, Antonio R. (1996). "Preparando un nuevo modo de conocer". Edutec. Revista de tecnología educativa, n. 4.

https://doi.org/10.21556/edutec.1996.4.573

Beck, Ulrich (1998). La sociedad del riesgo. Hacia una nueva modernidad. Barcelona: Paidós. ISBN: 9788449318924

Buckingham, David (2007). "Digital media literacies: Rethinking media education in the age of the internet". Research in comparative and international education, v. 2, n. 1, pp. 43-55.

https://doi.org/10.2304/rcie.2007.2.1.43

Burgess, Jean; Green, Joshua (2009). YouTube: Online video and participatory culture. Cambridge, UK: Polity Press. ISBN: 9780745644790

Butler, Judith (2007). El género en disputa. El feminismo y la subversión de la identidad. Barcelona: Paidós. ISBN 97884 49320309

http://www.lauragonzalez.com/TC/EI_genero_en_disputa_Buttler.pdf

Castells, Manuel (1997). La era de la información. Economía, sociedad y cultura. 3 vols. Madrid: Alianza. ISBN: 97884 20677002

Cavalcante, Andre (2016). "I did it all online': Transgender identity and the management of everyday life”. Critical studies in media communication, v. 33, n. 1, pp. 109-122.

https://doi.org/10.1080/15295036.2015.1129065

De-Ridder, Sander; Van-Bauwel, Sofie (2015). "Youth and intimate media cultures: Gender, sexuality, relationships, and desire as storytelling practices in social networking sites". Communications. The European journal of communication research, v. 40, n. 3, pp. 319-340.

https://doi.org/10.1515/commun-2015-0012

Ellison, Nicole; Boyd, Danah (2013). "Sociality through social network Sites". En: Dutton, William H. (ed.). The Oxford handbook of internet studies. ISBN: 9780199589074

https://doi.org/10.1093/oxfordhb/9780199589074.013.0008

Fraser, Nancy (1990). "Rethinking the public sphere: A contribution to the critique of actually existing democracy". Social text, n. 25/26, pp. 56-80.

https://doi.org/10.2307/466240

Giddens, Anthony (1995). Modernidad e identidad del yo. El yo y la sociedad en la época contemporánea. Barcelona: Península. ISBN: 8429737847

Habermas, Jürgen (1987). The structural transformation of the public sphere. An inquiry into a category of bourgeois society, 328 pp. The MIT Press. ISBN: 9780262581080

Halberstam, Jack (2018). Trans*. Una guía rápida y peculiar de la variabilidad de género. Barcelona-Madrid: Egales. ISBN: 9788417319328

Horak, Laura (2014). "Trans on YouTube intimacy, visibility, temporality". TSQ: Transgender studies quarterly, v. 4, n. 1, pp. 572-585.

https://doi.org/10.1215/23289252-2815255 
Jackson, Sarah J.; Foucault-Welles, Brooke (2015). “Hijacking \#myNYPD: Social media dissent and networked counterpublics". Journal of communication, v. 65, n. 6, pp. 932-952.

https://doi.org/10.1111/jcom.12185

Jenkins, Henry; Ito, Mizuko; Boyd, Danah (2016). Participatory culture in a networked era. Malden: Polity Press. ISBN: 9780745660707

Jenzen, Olu (2017). "Trans youth and social media: Moving between counterpublics and the wider web". Gender, place \& culture. A journal of feminist geography, v. 24, n. 11, pp. 1626-1641.

https://doi.org/10.1080/0966369X.2017.1396204

Jenzen, Olu; Karl, Irmi (2014). “Make, share, care: Social media and LGBTQ youth engagement". Ada: A journal of gender, new media, and technology, n. 5.

https://doi.org/10.7264/N39P2ZX3

Kellner, Douglas; Kim, Gooyong (2010). "YouTube, critical pedagogy, and media activism". Review of education, pedagogy and cultural studies, v. 32, n. 1, pp. 3-36.

https://doi.org/10.1080/10714410903482658

Masip, Pere; Ruiz-Caballero, Carlos; Suau, Jaume (2019). "Active audiences and social discussion on the digital public sphere. Review article”. El profesional de la información, v. 28, n. 2, e280204.

https://doi.org//10.3145/epi.2019.mar.04

Morduchowicz, Roxana (2012). Los adolescentes y las redes sociales. La construcción de la identidad juvenil en internet. Buenos Aires: Fondo de Cultura Económica. ISBN: 9789505579075

Papacharissi, Zizi (2002). "The virtual sphere: The internet as a public sphere”. New media \& society, v. 4, n. 1, pp. 9-27. https://doi.org/10.1177/14614440222226244

Papacharissi, Zizi (2010). A private sphere: Democracy in a digital age. Cambridge: Polity Press. ISBN: 9780745645254

Raun, Tobias (2010). "Screen-births: Exploring the transformative potential in trans video blogs on YouTube". Graduate journal of social science, v. 7, n. 2, pp. 113-130.

http://www.gjss.org/sites/default/files/issues/chapters/papers/Journal-07-02--07-Raun.pdf

Raun, Tobias (2016). Out online: trans self-representation and community building on YouTube. New York: Routledge. ISBN: 9781315599229

Renninger, Bryce (2014) “'Where I can be myself ... where I can speak my mind': Networked counterpublics in a polymedia environment". New media \& society, v. 17, n. 9, pp. 1513-1529.

https://doi.org/10.1177/1461444814530095

Rosen, Sam (2015). An act of violence: Transfemininity and the media. https://www.sarahlawrence.edu/faculty/media/Rosen_Transfemininity.pdf

Shifman, Limor (2011). “An anatomy of YouTube meme”. New media \& society, v. 14, n. 2, pp. 187-213. https://doi.org/10.1177/1461444811412160

Sibilia, Paula (2008). La intimidad como espectáculo. Buenos Aires: Fondo de Cultura Económica. ISBN: 9789505577545

Travers, Ann (2003). "Parallel subaltern feminist counterpublics in cyberspace”. Sociological perspectives, v. 46, n. 2, pp. 223-237.

https://doi.org/10.1525/sop.2003.46.2.223

Trejo-Delarbre, Raúl (2009). "Internet as an expression and extension of public space". Matrizes, v. 2, n. 2, pp. 1-16. https://doi.org/10.11606/issn.1982-8160.v2i2p71-92

Van-Zoonen, Liesbet (1994). Feminist media studies. London: Sage. ISBN 9780803985544

Wargo, Jon M. (2017). “'Every selfie tells a story...': LGBTQ youth lifestreams and new media narratives as connective identity texts". New media \& society, v. 19, n. 4, pp. 560-578.

https://doi.org/10.1177/1461444815612447

Warner, Michael (2002). "Publics and counterpublics". Public culture, v. 14, n. 1, pp. 49-90.

https://doi.org/10.1215/08992363-14-1-49 